\title{
Integrative analysis of the transcriptome profiles observed in type 1, type 2 and gestational diabetes mellitus reveals the role of inflammation
}

Adriane F Evangelista ${ }^{1}$, Cristhianna VA Collares ${ }^{1,2}$, Danilo J Xavier ${ }^{1}$, Claudia Macedo ${ }^{1}$, Fernanda S Manoel-Caetano ${ }^{1}$, Diane M Rassi ${ }^{1}$, Maria C Foss-Freitas ${ }^{2}$, Milton C Foss², Elza T Sakamoto-Hojo ${ }^{1,3}$, Catherine Nguyen ${ }^{4}$, Denis Puthier ${ }^{4}$, Geraldo A Passos ${ }^{1,5}$ and Eduardo A Donadi, ${ }^{1 *}$

\begin{abstract}
Background: Type 1 diabetes (T1D) is an autoimmune disease, while type 2 (T2D) and gestational diabetes (GDM) are considered metabolic disturbances. In a previous study evaluating the transcript profiling of peripheral mononuclear blood cells obtained from T1D, T2D and GDM patients we showed that the gene profile of T1D patients was closer to GDM than to T2D. To understand the influence of demographical, clinical, laboratory, pathogenetic and treatment features on the diabetes transcript profiling, we performed an analysis integrating these features with the gene expression profiles of the annotated genes included in databases containing information regarding GWAS and immune cell expression signatures.
\end{abstract}

Methods: Samples from 56 (19 T1D, 20 T2D, and 17 GDM) patients were hybridized to whole genome one-color Agilent $4 \times 44 \mathrm{k}$ microarrays. Non-informative genes were filtered by partitioning, and differentially expressed genes were obtained by rank product analysis. Functional analyses were carried out using the DAVID database, and module maps were constructed using the Genomica tool.

Results: The functional analyses were able to discriminate between T1D and GDM patients based on genes involved in inflammation. Module maps of differentially expressed genes revealed that modulated genes: i) exhibited transcription profiles typical of macrophage and dendritic cells; ii) had been previously associated with diabetic complications by association and by meta-analysis studies, and iii) were influenced by disease duration, obesity, number of gestations, glucose serum levels and the use of medications, such as metformin.

Conclusion: This is the first module map study to show the influence of epidemiological, clinical, laboratory, immunopathogenic and treatment features on the transcription profiles of T1D, T2D and GDM patients.

Keywords: Type 1 diabetes, Type 2 diabetes, Gestational diabetes, Module map, Immune cell signatures, Transcriptome analysis

\footnotetext{
* Correspondence: eadonadi@fmrp.usp.br

${ }^{1}$ Molecular Immunogenetics Group, Department of Genetics, Faculty of

Medicine of Ribeirão Preto, University of São Paulo (USP), 14049-900 Ribeirão

Preto, SP, Brazil

${ }^{2}$ Division Clinical Immunology, Faculty of Medicine of Ribeirão Preto, (USP),

14049-900 Ribeirão Preto, SP, Brazil

Full list of author information is available at the end of the article
} 


\section{Background}

Diabetes mellitus is considered by the World Health Organization to be a global epidemic. Although the different types of diabetes have varying incidence rates, it is estimated that approximately $6.4 \%$ of the world adult population have some type of diabetes [1]. Diabetes is characterized as hyperglycemia resulting from a relative or absolute impairment of insulin secretion as well as peripheral resistance to insulin [2]. Based on etiology, diabetes mellitus has been classified into type 1 diabetes (T1D), type 2 diabetes (T2D), gestational diabetes mellitus (GDM), as well as other types of diabetes, including genetic defects in $\beta$-cell function, genetic defects in insulin action, diseases of the exocrine pancreas, endocrinopathies, drug- or chemical-induced forms, infection-induced diabetes, uncommon forms of immunemediated diabetes, and other genetic syndromes associated with diabetes [2]. The pathogenic mechanisms of each diabetes type are still unclear, especially in the case of T2D and GDM. Pregnant women with gestational diabetes also have an increased risk of developing T2D, suggesting a close relationship between these types [3,4].

T1D is the most studied type of diabetes, has several susceptibility loci identified in mice (Idd1-Idd26) and is characterized by the autoimmune destruction of pancreatic beta cells leading to insulin deficiency [5]. Macrophages, dendritic cells and lymphocytes are involved in this pathogenic process through a complex interplay of mechanisms implicated in the loss of immune tolerance to autoantigens, including i) the hypoexpression of insulin in the thymus during promiscuous antigen expression [5,6]; ii) autoantigen presentation mediated by molecules coded by the HLA-DRB1*04-DQB1:03:02 and $H L A-D R 31 * 03-D Q B 1 * 02: 01$ haplotypes resulting in the development of insulin autoantibodies (IAA) and autoantibodies against the $65 \mathrm{kDa}$ isoform of glutamic acid decarboxylase (GADA), respectively [7]; iii) a deregulation of the immune response mediated by either an impaired expression of surface regulatory molecules (IL2RA, IL2RB, and CTLA-4) or a deregulation of intracellular signals (PTPN2 and PTPN22) [8]; iv) a decreased number of suppressive or T regulatory cells [9]; v) a decreased number of iNKT cells [10]; and vi) a loss of function of molecules involved in the innate immune response [11,12]. In addition, several other genes have been implicated in the development of T1D by human genome wide association studies (GWAS) [13-15], including genes identified by transcriptome analyses in human or animal models $[16,17]$ that evaluated peripheral blood mononuclear cells $[18,19]$, pancreatic beta cells [20] and whole blood cells [21].

In recent years, genetic mechanisms have also been shown to affect the development of T2D [22]. Several studies have associated polymorphisms in the PPARG and $K C N J 11$ genes with T2D susceptibility [23]. Other studies have identified a strong effect of TCF7L2 gene variants on T2D risk, possibly affecting proglucagon expression with consequent reduced insulin secretion [23,24]. Indeed, both GWAS and international collaborative efforts to analyze GWAS data from multiple groups, such as the Meta-Analysis of Glucose and Insulin-related traits Consortium (MAGIC), have identified other genetic variants associated with T2D gene susceptibility $[25,26]$, several of which were associated with glycemic traits. Many of these groups of genes were related to abnormal insulin processing $(M A D D)$, higher proinsulin and lower insulin secretion (TCF7L2, SLC3OA8, GIPR, and C2CD4B), and abnormalities in early insulin secretion (MTNR1B, FADS1, $D G K B$, and $G C K)$ [22]. Although GWAS have identified susceptibility regions across the genome and transcriptome studies have indicated several modulated genes in beta cells and the usefulness of blood RNA profiles [27,28], functional studies are still needed to understand the role of several genes obtained from association studies.

The pathogenesis of T1D has been considered to be different from that of T2D, and information obtained by GWAS has indicated that most T1D and T2D genetic loci seem to not overlap. However, there is evidence that inflammatory processes involving interleukin- 1 may play a role in islet beta cell loss in both types of diabetes [29]. Peripheral blood mononuclear cell (PBMC) energy metabolism has been found to play a major role in the pathogenesis of insulin resistance [30]. In addition, there is increasing evidence that metabolic regulation in these cells could influence the number of PBMCs, proliferation pathways, molecular basal synthesis and leukocyte function [31]. In accordance with these findings, transcriptome studies comparing T1D with T2D [32] and T1D with a control group [18] have revealed changes in the expression of several genes related to inflammatory response, fatty acid biosynthesis, hydrolase activity, detoxification of aldehydes generated by alcohol metabolism and lipid peroxidation, all of which could affect the metabolism of PBMCs in diabetic patients.

Compared with T1D and T2D, GDM has been subject to fewer linkage studies and transcriptome analyses [33]. Many genes, however, are known to be associated with both GDM and T2D, especially those related to obesity and oxidative stress $[34,35]$. The mechanisms linking excess adiposity to an elevated risk of GDM are not completely understood, but recent evidence points to the role of specific hormones and cytokines known as adipokines, which are secreted by the adipose tissue $[36,37]$. In addition, transcriptome signatures obtained from placenta [38] and whole blood cells [39] have identified genes involved with lipid metabolism that are differentially expressed between T1D and GDM. Recently, in a meta-analysis of the transcription profiles of T1D, T2D and GDM patients, our group reported that gene expression signatures of GDM patients were closer to those of 
T1D patients than to T2D [40]. The analyses of gene expression signatures, however, were impaired by the presence of multiple variables associated with each type of diabetes. To circumvent these problems, here we used several bioinformatics tools to analyze demographical, clinical, laboratory, pathogenetic and treatment data against modulated genes that have been annotated by databases containing information regarding both GWAS and gene expression signatures displayed by immune cells. This type of comparison has yielded multiple informative modules that have been used to perform comprehensive maps in cancer [41], and here we apply these tools to diabetes.

\section{Results}

Overall, a schematic heatmap with all demographic, clinical and laboratory patient features is shown in Figure 1. The global partitioning analysis of the three types of diabetes disclosed 8,469 transcripts considered as informative, which are available at www.rge.fmrp.usp.br/passos/DBF-MCL, the principal component analysis (PCA) of these genes are shown in Figure 2 and the summarized DAVID functional categories (Kegg pathways) of the main clusters are shown in Figure 3.

The Venn diagrams yielded shared and specific genes after statistical analysis by rank products (T1D versus T2D, T2D versus GDM and T1D versus GDM) (Figure 4) as well as multiple significant summarized DAVID functional categories (Kegg pathways) (Figure 5). The module maps encompassing all analyses, i.e., genes obtained from both partitioning and rank products, were created with the set of genes obtained in each of the approaches described above (Figure 6). Finally, the confirmation by PCR analysis of important genes involved in diabetes is shown in Figure 7.

Global analysis of the data set with density-based filter and Markov clustering (DBF-MCL) and principal component analysis (PCA)

A list of 8,469 genes obtained from global partitioning analysis (DBF-MCL method applying a non-informative filter) yielded informative non-supervised clusters of

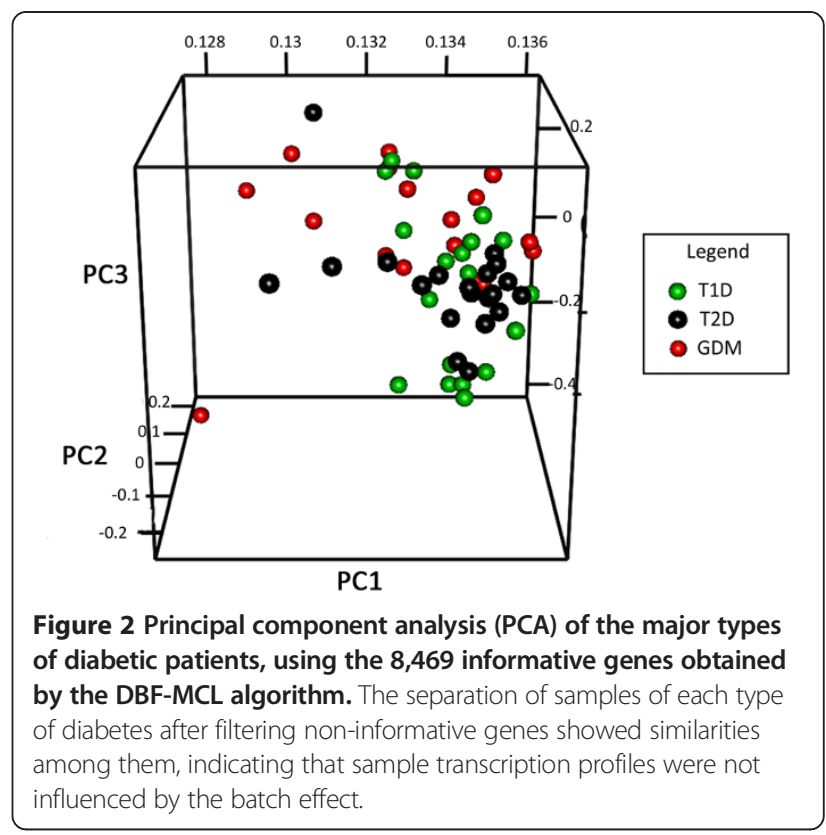

co-regulated genes. Figures showing all non-supervised clusters and the respective gene list are available at www.rge.fmrp.usp.br/passos/DBF-MCL. A PCA plot of filtered genes is shown (Figure 2). Despite we have more similarity between T1D and GDM, some patients (with the lowest levels of glycemic levels) were closer to the other types. Overall, many gene expression profiles are shared between T1D and GDM. A typical example of this analysis can be observed in cluster \#1 (3,089 probes), in which all T2D patients shared a group of induced genes encoding zinc finger proteins, while these same genes were found repressed in T1D and GDM patients. Similarly, in cluster \#12 (216 probes), all T2D patients shared a group of repressed genes associated with cytokine and chemokine activity, NOD-like and Toll-like receptor signaling pathways and MAPK signaling pathway (Figure 3).

\section{Supervised analysis of T1D, T2D and GDM patients}

To understand the influence of demographic, clinical, pathogenetic and laboratory features on the differential

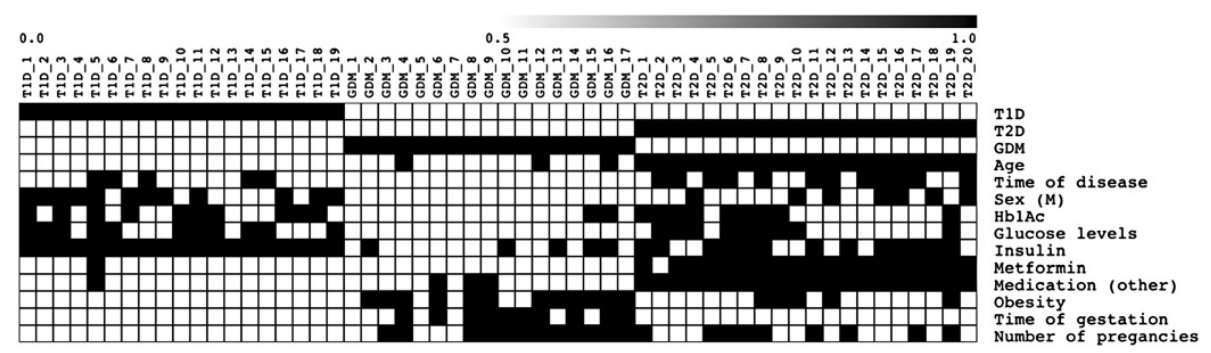

Figure 1 Heatmap representative of type of diabetes, demographic, clinical, laboratory and treatment features of the patients.

Qualitative variables were assigned by the absence or presence of the characteristic, and quantitative variables were assigned by values below or above the mean values. This information was used as array (experimental) set for the module map construction. 


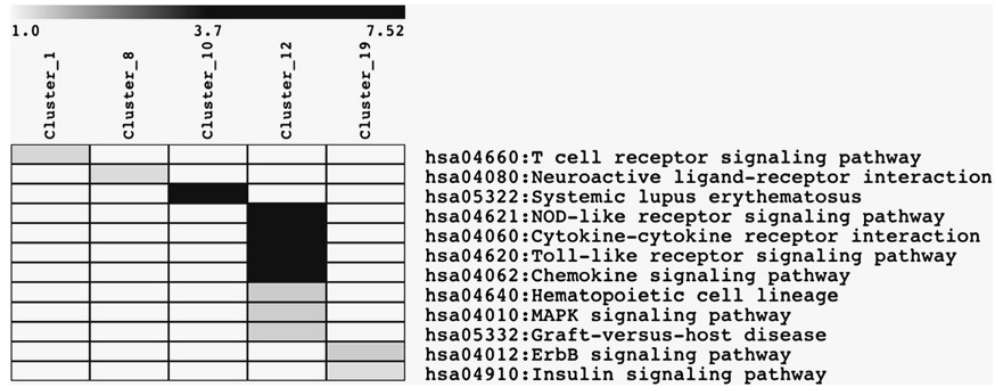

Figure 3 Heatmap of the significant functional categories of the genes of the clusters obtained by non-informative filters, in the basis of a FDR $\leq \mathbf{0 . 1}$. The Kegg categories were obtained from DAVID knowledge base with an enrichment $P$ value $\leq 0.05$ after Benjamini correction. The grey scale represents the logarithm of the enriched $P$ value.

gene expression profiles among diabetes patients, a second strategy was used. Module maps were created by comparing the three groups at the same time (from the partitioning analysis) as well as by the individual two by two group comparisons. These paired analysis module maps were constructed based on the results obtained from statistical analyses of rank products and are represented in Venn diagrams (Figure 4), disclosing up and down-regulated genes shared by the same type of diabetes in different analysis. The functional categories of these genes are shown in Figure 5.

T1D patients presented several genes that were induced when compared to GDM patients, including genes in the MHC region (HLA-DQA1 and HLA-DQA2), TNF receptors (TNFRSF17, TNFAIP6), cytokines (IL1A, IL-1B, IL1F10, IL4, IL6, IL8, IL23A, IL27) and cytokine receptors (IL1R2, IL1R1N, IL18R1), chemokines (CXCL1, CXCL2, CCL20, CCL23, CCL3L3, CCL4) and chemokine receptors (CCR3, CRL2), lymphocyte receptors (KIR3DL2, KIR2DS4), and transcription factors (GLIS2, SOX8, GATA2, RUNX1, SOD2, FOXC1, FOXC2, FOXE1). Additionally, T1D patients presented several genes that were differentially expressed compared to T2D patients, including immune response genes, as $\mathrm{MHC}$ region genes (HLA-DQA1 and $D Q A 2)$, clusters of differentiation genes $(C D 8 B, C D 55$, CD83), cytokines (IL1A, IL1B,IL6, IL8, IL23A), chemokines (CXCL1, CCL3L3, CXCL2, CXCL3, CCL20, CCL23, CCL24, $C C L 3, C C L 4)$, NOD-like receptor signaling pathway (CXCL1, IL6, CARD9, TNF, IL8, CXCL2, CASP8, NFKBIA, IL1B, MAPK8, TNFAIP3, NLRP3) and transcription factors (GLIS2, SOX8, FOXC2, FOXC1, FOXE1, FOXK1).

GDM patients presented several genes that were induced compared to T1D patients, including toll-like receptors (TLR6, TLR7), carbohydrate binding genes (LGALS3, $L G A L S 12, C L E C 7 A, C L E C 1 B)$, plasma membrane receptors (IL5RA, CCR1), MHC genes (HLA-DRB3), solute carrier family genes (SLC6A10P, SLC6A4, SLC1A5, SLC4A1, SLC8A1, SLC6A17, SLC16A3, SLC6A8, SLC14A1), cluster of differentiation genes (CD9, CD33, CD36), and chemokines (CXCL5, CCL15, CXCL12, CXCL10). In contrast, GDM patients presented several genes that were induced compared to T2D, including immune response genes (CXCL10, CXCR4, CD46, CCL3L3, IL1B, IL1A, NFKBIZ, IL27, C1QA, CD83, C1QB, HIF1A, DEFA4, DEFA3, KIR2DL4, CXCL1, CCL3, TNF, CCR1, CXCL3, CXCL2, CCL4, CCL23, CCL20, IL6, CR2, IL8, IL1RN STAT3,
A

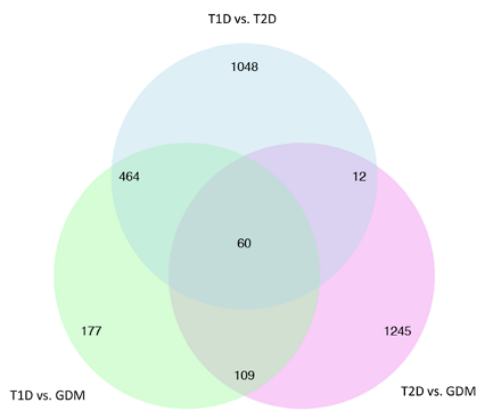

B

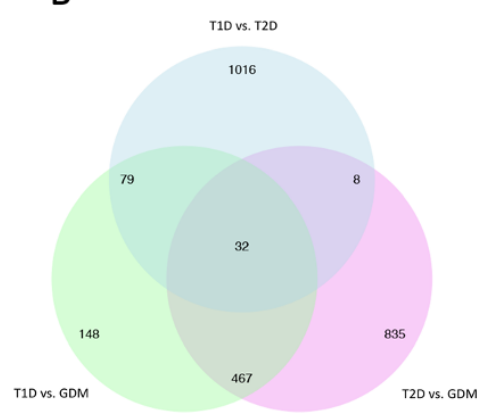

Figure 4 Venn diagrams show the differentially expressed genes after paired analysis of the three types of DM. The genes were identified by Rank Product analysis with $P$ value $\leq 0.001$ and a percentage of false prediction (pfp) $\leq 0.05$. The analysis referring to upregulated genes is shown in panel $\mathbf{A}$ and that of downregulated genes in panel $\mathbf{B}$. 


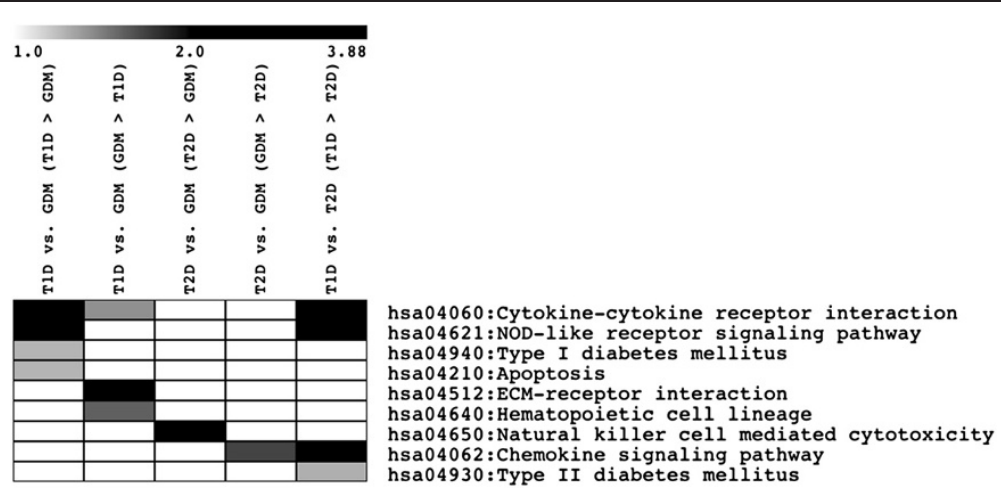

Figure 5 Heatmap of the significative functional categories of the differentially expressed genes obtained by paired Rank Products analysis with $P$ value $\leq 0.001$ and percentage of false prediction (pfp) $\leq 0.05$ (T1D vs. GDM; T2D vs. GDM and T1D vs. T2D). The Kegg categories were obtained from DAVID knowledge base with an enrichment pvalue $\leq 0.05$ after Benjamini correction. The grey scale represents the logarithm of the enriched $P$ value.

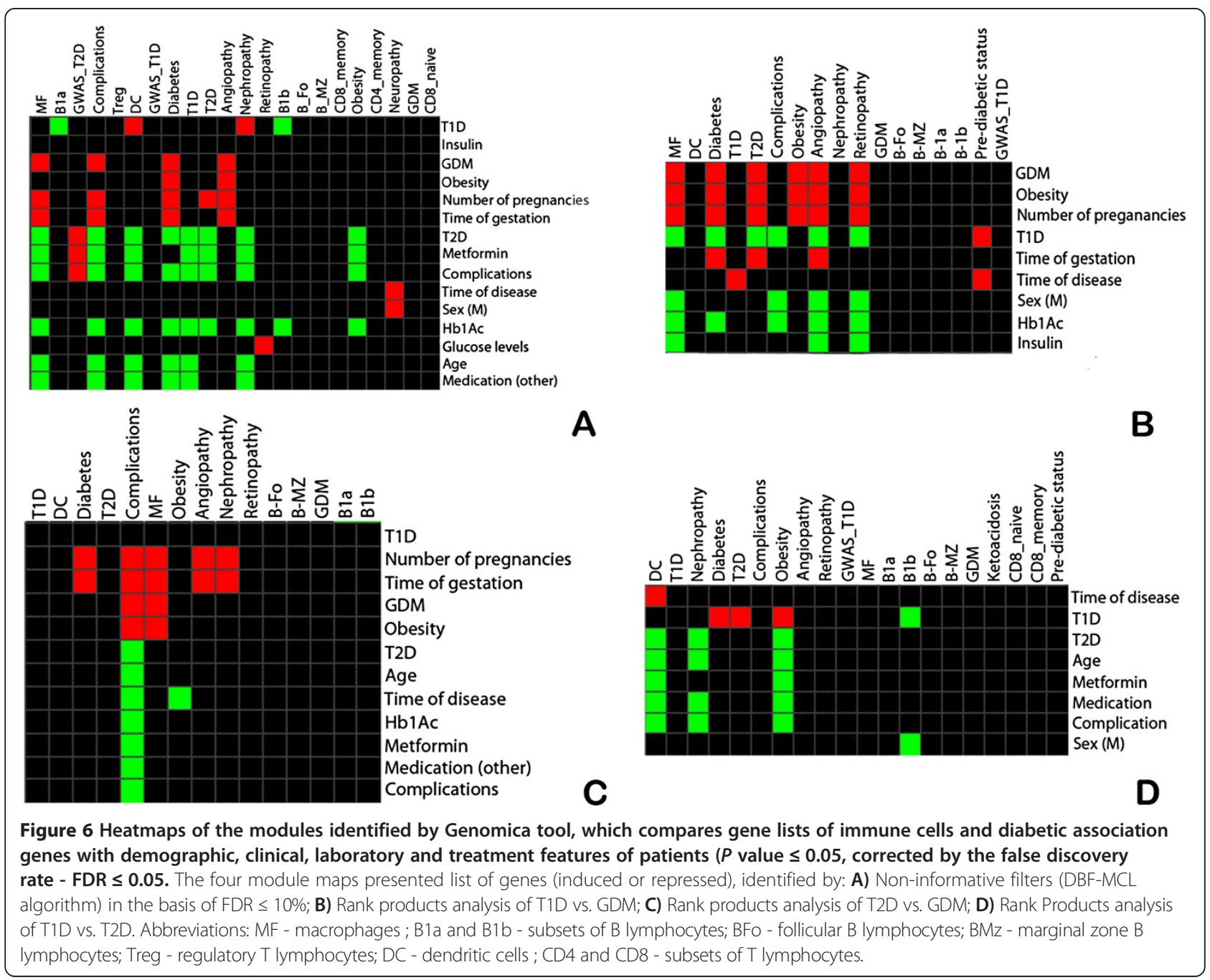




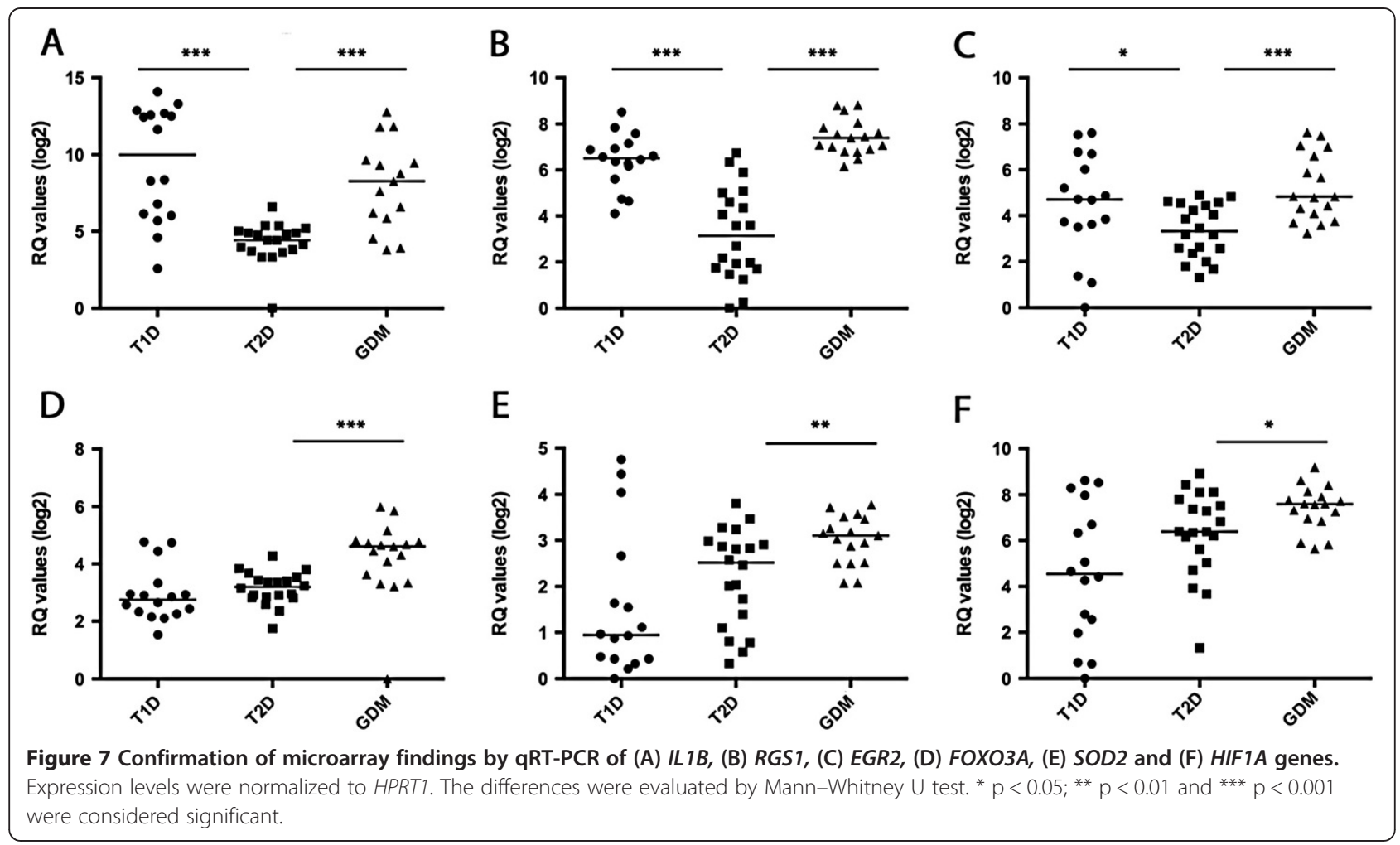

$C D 55)$ and genes involved in the response to hormone stimulus (PTGS2, LDLR, PGF, PDGFA, PTGS1, NOS3, ADAM9, EGR2, SOCS2, SOCS3, RXRA, ADIPOR1, GAL, JUNB, C1QB, RETN, ADM, SORT1, CAV2, CAV1, TNF, GRB2, ERBB3, GNG11, BCL2L1, PIK3R1, IL6, IL1RN, STAT1, STAT3, CDKN1A, BMP7, IGFBP2).

T2D patients presented several genes that were differentially expressed compared to T1D patients, including genes of numerous zinc finger proteins and genes regulating transcription (ZNF184, ZNF576, ZNF449, ZNF594, ZNF641, ZNF100, ZNF644, ZNF189, ZNF785, ZNF436, ZNF613, among others). Finally, the comparison between T2D and GDM showed multiple genes induced in T2D patients, including genes involved in DNA-binding, as zinc finger (ZNF582, ZNF250, ZNF184, ZNF181, ZNF775, ZNF773, ZNF248, ZNF397, ZNF578, ZNF442, ZNF443, among others).

\section{Comprehensive functional analysis using a module map approach}

To identify the influence of patient features (array or experimental sets) on gene information (gene sets), we constructed several module maps, stratifying patients according to demographic, clinical, laboratory and therapeutic characteristics. We used specialized databases associated with diabetes complications [42], gene clusters associated with diabetes obtained from association studies (GWAS) [43], and isolated immune cell types associated with the pathogenesis of diabetes [44]. The most relevant modules are shown in Figure 6.

GDM patients exhibited up-regulated genes observed in diabetes complications (including angiopathy) and in macrophages. GDM, number of gestations per patient and gestation time were associated with the induction of diabetic complications genes. Interestingly, a history of 2 or more gestations was positively associated with the modulation of T2D genes, while in T1D patients the number of gestations did no influence the transcription profile of female patients (Phenopedia). T1D patients exhibited induced genes typical of those displayed by dendritic cells and repressed genes typical of those presented by Blymphocytes. In addition, T1D patients exhibited induction of genes related to diabetes nephropathy. The use of insulin did not influence gene expression patterns; however, increased serum glucose level was associated with the induction of genes related to diabetic retinopathy. In patients with T2D, the disease itself as well as the use of metformin was associated with the repression of genes implicated in obesity and diabetic complications. In contrast, genes found by T2D GWAS were associated with genes induced in T2D patients and the use of metformin. Male patients who had T2D for 11 or more years were at increased risk of neuropathy. The heatmap shown in Figure 6A illustrates these results.

In the paired analysis of T1D and GDM patients, both obesity and a history of two or more gestations were 
positively associated with T2D development. Conversely, in T1D patients, these variables were negatively associated. Additionally, in T1D patients, the use of insulin repressed genes of angiopathy and retinopathy, and macrophage gene expression was associated with GDM (Figure 6B). In the paired analysis of T2D and GDM, the number of gestations was positively associated with genes related to diabetic complications. The use of metformin was negatively associated with complications, and these same genes were repressed in T2D (Figure 6C). The paired analysis of T1D and T2D patients showed repression of dendritic cell genes associated with T2D and the induction of dendritic cell genes associated with time of disease. Obesity genes were also induced in T1D patients (Figure 6D).

\section{Confirmation by real-time PCR}

We performed the confirmation of EGR2, RGS1, FOXO3A, HIF $1 A, I L 1 B$ and SOD2 genes (Figure 7), which were chosen because some of these genes have been previously described as specific for GDM compared with T2D (SOD2, FOXO3A, HIF1A). Compared to GDM, these genes were downregulated in T2D as evaluated by microarrays and qRT-PCR, presenting fold changes of 4.34, 1.75 and 1.72, respectively (microarrays), and 1.51, 2.66 and 2.31 , respectively (qRT-PCR). In addition, compared to T2D, the EGR2, IL1B and RGS1 genes were downregulated when compared to T1D (fold changes of 1.82, 13.96 and 3.08, respectively, for microarrays and $2.59,47.83$, and 9.95, respectively, for qRT-PCR), and downregulated when compared to GDM (fold changes of 2.22, 11.11 and 4.34, respectively, for microarrays, and 2.82, 14.50, and 18.36, respectively, for qRT-PCR).

\section{Discussion}

Diabetes mellitus is one of the most studied diseases, and a large amount of information is available in the public databases regarding genetic association, meta-analysis and associated complications. Few studies, however, have systematically compared the major types of diabetes in terms of gene expression profiles at the genomic level. In this study, we performed an integrative analysis of the transcriptome profiles of the major types of diabetes using several bioinformatics tools. The major innovation of this study was the construction of informative module maps integrating epidemiologic, clinical, laboratory, pathogenetic, genetic, and molecular factors implicated in diabetes to identify individual and shared features in T1D, T2D and GDM.

The major finding of our global partitioning analysis that examined the differences in transcription among each diabetes patient was the cluster of genes associated with inflammation. The high expression levels of these genes in some T1D and GDM patients seem to influence the global gene expression pattern of diabetic patients. Indeed, several important molecular mechanisms identified by clustering account for an intricate array of inflammation pathways. One important finding was the up-regulation of many mediators of the NOD-like receptor signaling pathway. It has recently been suggested that NOD-like receptors can be induced by hyperglycemia and oxidative stress products, which could link metabolism and inflammation, particularly through the participation of $I L 1 B$ [45]. In this study, many genes involved in these pathways were induced in T1D and GDM patients, including $N L R P 3$ (an important receptor of the NOD-like pathway), IL1B, CXCL1, CXCL2, IL6, IL8, TNF, RIPK2, TNFAIP3 and NFKB1A. TNF and IL1B are strongly involved in the regulation of nitric oxide biosynthetic processes [46] and are regulated by SOD2 [47]. In addition, other upregulated genes including chemokines (CCL3, CCL4), cytokines (IL6, IL8, TNF) and transcription factors (NFKBIA, MAPK8) are involved with other inflammatory processes, such as toll-like receptors signaling pathway, and the expression of $I L 1 B$ and SOD2 genes was confirmed by real-time PCR. Curiously, genes associated with these processes were downregulated in T2D compared with the other types of diabetes. To understand this result, we took advantage of the rank product analysis (paired analyses), which showed particular features of the involvement of these inflammatory pathways in each type of diabetes. The comparisons between T2D and GDM as well as between T1D and T2D revealed several interesting results. At the same time that NOD-like receptors were induced in T1D and GDM, hundreds of zinc finger protein genes were induced in T2D. Additionally, drug treatment with metformin in T2D patients seems to influence the gene expression patterns, whereas insulin treatment did not. As literature findings have indicated that the expression of transcription factors associated with T2D can be induced by hypoglycemiants, it is possible to hypothesize that these drugs may induce alterations in the normal T2D expression profile. Finally, the comparisons between T2D and T1D reveal the repression of important genes associated with T2D diabetes, particularly transcription factors involved in glucose homeostasis (TCF7L2) [24,48], $\mathrm{NAD}+($ FOXO3) $[49]$ and regulation of cellular and systemic response to hypoxia (HIF1A) [50], and the differential expression of HIF1A and FOXO3A in our series was also confirmed by real-time PCR.

To further understand the close similarity between the transcription profiles in T1D and GDM, we examined the paired comparisons between these types. T1D patients exhibited modulation of genes in the MHC region, including the induction of $H L A-D Q A 1$ and $H L A-D Q A 2$. The $D Q A$ gene encodes the alpha chain of the HLA-DQ heterodimeric molecule. HLA-DQ is non-covalently associated with $\mathrm{DQ} \beta$, which has been associated with T1D 
susceptibility in multiple populations [51-54]. In contrast, few studies have associated any HLA class II genes, including HLA-DRB3 genes, induced in GDM [53]. Although a role of HLA class II genes in T1D pathogenesis has not yet been established, decreased surface expression of HLA-DQ molecules on CD4 and CD8 peripheral cells has been reported in recently diagnosed T1D patients exhibiting $D Q B 1$ susceptibility alleles. This finding was attributed to the instability of these molecules on the cell surface [54]. As none of our patients were recently diagnosed, it is interesting to observe that the expression of $D Q A$ MHC molecules is still modulated even after the disease has been present for long periods of time. On the contrary, there is also evidence that humoral responses to autoantigens may be driven by the $H L A-D Q A 1$ genes even in recently diagnosed patients [55]. MHC susceptibility alleles associated with T1D (DQA1*05:01, DQB1*02:01/DQB1*03:02) are different from those associated with GDM [56]. In addition to MHC genes, T1D patients also showed different levels of the killer immunoglobulin-like receptor (KIR3DL2 and KIR2DS4) family genes compared with GDM patients (KIR2DL4). There are no gene expression studies implicating KIRs in diabetes. These findings suggest that the close similarity of the T1D and GDM transcription profiles may be due to the overall inflammatory gene patterns observed in both conditions, including the modulation of several genes primarily involved with the innate immune response.

Previously, the construction of module maps for cancer patients has revealed important modules that characterize different cancer lineages [41], allowing the identification of cancer biomarkers. Major findings regarding each group of diabetes patients are discussed below, including epidemiological, clinical, laboratory, genetic and pathogenetic features. In all module maps in which the gene profile of GDM was compared with other types of diabetes, we observed an up-regulation of genes typically expressed by macrophages. Overall, the comparison with genesets with arraysets yielded positive associations, except for T1D, which may be attributed to differences in patient age and the autoimmune nature of T1D. These macrophage genes were co-regulated with those appearing in diabetic complications such as angiopathy and retinopathy, including the ILIB and RGS1 genes, as seen in Figure 6A, B and C. As we also observed that increased glucose levels were associated with the development of retinopathy, it is possible that the effect of high glucose levels on macrophages might be involved in the creation of GDM complications. Moreover, GDM patients exhibited gene profiles similar to those reported for obesity (data available in the Phenopedia public data banks), and the majority of patients in our study were overweight. It is also interesting to observe that obesity, two or more gestations per patient, and gestations lasting over 30 weeks exhibited the same modules of induced genes in GDM patients, as observed in Figure 6A. Additionally, in GDM patients, having two or more gestations was positively associated with the development of T2D. Indeed, it is important to note that obese GDM patients have an increased risk of developing T2D [57]. This information is important for future studies of GDM.

In T1D patients, we observed a positive association with the profile reported for dendritic cells (ImmGen) (Figure 6A), particularly in patients exhibiting long-term disease (Figure 6D). As dendritic cells play an important role in antigen presentation via MHC class II molecules and as MHC class II genes are also induced in long-term T1D patients, it is possible to hypothesize that abnormal antigen presentation (foreign or self) is a chronic phenomenon in T1D. In contrast, T1D patients also exhibited a repression of genes associated with $\mathrm{B} 1 \mathrm{a}$ and $\mathrm{B} 1 \mathrm{~b}$ lymphocytes (naive B cells), in which the B1a lymphocyte subtype (CD5+) has been associated with the production of natural and autoantibodies [58]. Unfortunately, until the moment, there is no public data regarding the gene pattern of activated B cells. Another important finding is the similarity of T1D gene expression profiles with that observed for diabetes nephropathy (Phenopedia). As no patients in our analysis exhibited clinical nephropathy, it may be valuable to further study the particular patients exhibiting nephropathy related gene patterns.

Finally, T2D patients exhibited gene expression profiles that were in disagreement with those reported in the public databases (Phenopedia) for diabetic complications (Figure 6C). Considering that half of our T2D patients exhibited higher median glucose levels than our T1D and GDM patients, one could expect to find the induction of genes associated with diabetic complications. As the T2D patients were treated with many types of medications besides hypoglycemiants, it is possible that this intriguing and unforeseen finding may be a consequence of these treatments. Another possible explanation may be related to inflammation genes, which were downregulated in T2D compared to T1D and GDM patients. The coupling of inflammation and drugs used to treat T2D might be responsible based on these findings. Indeed, the use of metformin and other medications (aspirin, captopril, atorvastatin, and hydrochlorothiazide) seems to modulate the expression of a large number of genes (Figure $6 \mathrm{C}$ and D), possibly affecting the inflammation status of T2D patients. Some of the down-regulated genes observed in T2D patients being treated with several drugs included IL1B, IL4, IL8, CCL2 and TNF. All of these genes are involved in inflammation and are also modulated in macrophages (data not shown). However, we cannot disregard the participation of the inflammatory pathway in T2D, as 
genes that participate in the NOD-like receptors signaling pathway probably also play a role in T2D [45]. Recent findings have shown that the down-regulation of FOXO1 expression in macrophages blocks lipid accumulation in these cells, affecting many processes [59]. In our analysis, FOXO3A was also down-regulated in T2D patients compared to GDM. Both FOXO1A and FOXO3A genes are mediators of the same signaling pathway, AMPK, which can be activated as a consequence of long-term metformin use [49], and the expression of FOXO3A was confirmed by real-time PCR.

In conclusion, our analysis revealed that $\mathrm{T} 1 \mathrm{D}$ and GDM exhibited a similar up-regulation of inflammatory genes.

\section{Methods}

\section{Study population}

We studied 56 adult diabetic patients, 19 presenting T1D (7 women/12 men) with ages of 18-36 years, 20 presenting T2D (13 women/7 men) with ages of 41-72 years and 17 presenting gestational diabetes with ages of 23 to 40 years. For our analysis, we used the mean age of the three groups (37 years with $\mathrm{SD} \pm 14$ ). The mean length of disease was $11 \pm 5.3$ years, while the mean values of glucose and Hb1Ac levels were $130.7 \mathrm{mg} / \mathrm{dL}$ with $\mathrm{SD} \pm 75.4$ and 8.9 with $\mathrm{SD} \pm 1.8$, respectively. For gestational diabetes patients, the mean period of gestation was 30.5 with SD \pm 5.7 weeks, and the mean number of pregnancies was $2 \pm 1$ per patient. Twelve GDM women had more than two gestations, while 9 T2D and none T1D women presented this characteristic. T1D and GDM patients were treated only with insulin, while T2D patients were treated with insulin in combination with metformin, captopril, aspirin, atorvastatin and hydrochlorothiazide. All patients underwent follow-up examinations at the Outpatient Clinics of the Division of Endocrinology, Faculty of Medicine of Ribeirão Preto, University of Sao Paulo, Brazil. The exclusion criteria were based on recent episodes of ketoacidosis, active nephropathy, proliferative retinopathy, diabetic foot syndrome, high LDL levels and diagnosed cardiovascular diseases. Figure 1 shows a schematic heatmap with all demographic, clinical and laboratory patient features. The study protocol was approved by the local Ethics Committee (Comitê de Ética em Pesquisa do Hospital das Clínicas e da Faculdade de Medicina de Ribeirão Preto da Universidade de São Paulo, under the permit \# 9153/2008), and informed consent was obtained from all participants.

\section{Blood collection, peripheral mononuclear cell isolation and RNA extraction}

A total of $20 \mathrm{~mL}$ of peripheral blood was collected and used for the isolation of PBMCs by discontinuous gradient density centrifugation on a Ficoll-Hypaque cushion
(Sigma, St. Louis, MO). Total RNA was extracted using the Trizol reagent (Invitrogen, Carlsbad, CA) according to the manufacturer's instructions. RNA concentrations and ratios were checked using a NanoDrop ND-1000 spectrophotometer (NanoDrop Products, Wilmington, $\mathrm{DE}$ ), and the RNA integrity was assessed by microfluidic electrophoresis using a 2100 Bioanalyzer and RNA 6000 nanochips (Agilent Technologies, Santa Clara, CA). We used only samples that exhibited median RNA integrity number $(\mathrm{RIN}) \geq 9.0$.

\section{Oligo microarrays}

Hybridizations onto whole human genome $4 \times 44 \mathrm{~K}$ oligo microarrays (G4112F, Agilent) were performed using the one color (Cy3) Quick Amp labeling kit (Agilent). Briefly, 500 ng of total PBMC RNA plus spike-in controls were reverse transcribed into double stranded cDNA. The primers used for this reaction contained many consecutive thymine bases attached to a $\mathrm{T} 7$ promoter that paired at the 5' end of the first strand of cDNAs. Next, the T7 polymerase was added along with nucleotides labeled with fluorescent cyanine-3 (Cy3) dye, which amplified the anti-sense complementary RNAs (cRNA). The cRNAs were purified and then hybridized to the microarray for 17 hours at $65^{\circ} \mathrm{C}$. After washing, the slides were scanned using a DNA Microarray Scanner with Surescan HighResolution Technology (Agilent). A complete file providing microarray data from all samples used in this study, as well as the numerical quantitative data and experimental conditions, is available on line at the ArrayExpress public database [60] through the following accession numbers: T1D (E-MEXP-3348), T2D (E-MEXP-3287) and GDM (E-MEXP-3349). These data correspond to a part of our laboratory databank, which were made publicly available and can be used for further studies and/or reanalysis.

\section{Data quantification and normalization}

Data quantification and quality control were analyzed using the Feature Extraction (FE) software version 10.7 (Agilent Technologies). Expression data were loaded into an R-environment [61] using the AgiND package [62], a tool developed by the Technologies Avancées pour le Genome et la Clinique (INSERM U1090, Marseille, France). The AgiND tool is available by request [63]. The background adjustment was performed by subtracting median background values from the median expression values obtained by $\mathrm{FE}$ and then converting the results to log-scale. For each sample, any negative values observed were replaced by randomly selected small positive values. After log-transformation, quantile normalization using the normalizeQuantile function was performed. 


\section{Density based filtering and Markov clustering (DBF-MCL) and principal component analysis (PCA)}

The Density Based Filtering and Markov Clustering (DBF$\mathrm{MCL}$ ) algorithm implemented in the Rtools4TB bioconductor package $[64,65]$ was used to extract sets of co-regulated genes from our microarray dataset. DBFMCL is a tree-step adaptive algorithm that finds genomic elements and genes located in dense areas, uses selected items to construct a graph and finally creates a partitioning graph using the Markov Clustering Algorithm (MCL). A $10 \%$ false discovery rate (FDR) was used. The principal component analysis (PCA) was performed from the informative genes identified by DBF-MCL. In this analysis, the R function prcomp [66] was used to evaluate PCA and the rgl package [67] to construct 3D graphics.

\section{Statistical analysis}

Differentially expressed genes were identified using the $\mathrm{R}$ package RankProd [68,69]. Although the non-parametric rank product method does not make any assumptions about the data distribution, it can provide frequency ranking scores at each data point and is thus a robust tool for creating ranking lists. Genes were considered significantly expressed when they presented p-values and percentage of false positive predictions (PFP) smaller than 0.001 and 0.05 , respectively. This test was used to perform paired analysis between T1D versus GDM, T2D versus GDM and T1D versus T2D. Venn diagrams illustrating up and downregulated genes in each analysis are shown in Figure 4.

\section{Module-map construction}

Significant and differentially expressed genes obtained by the use of DBF-MCL and Rank Products were initially clustered using the Cluster 3.0 and TreeView softwares [70,71]. Then, module maps were constructed using an ensemble of tools provided by the Genomica software [41], which searches for higher-order modules of gene sets and samples. Initially, this algorithm uses gene sets to annotate genes that were up-regulated (or down-regulated) by at least 1.5 -fold. The algorithm compares the modulated genes with array sets, including groups of compartmentalized genes, and organizes them into modules (module maps) discriminating variablespecific gene patterns according to patient features. It was used $P$ value $\leq 0.05$ with false discovery ration $(\mathrm{FDR}) \leq 0.05$.

\section{Module map array set variables}

The variables used to create the experimental sets included demographic parameters (age and gender), clinical variables (disease duration, obesity, duration and number of gestations), laboratory data (serum glucose and glycated hemoglobin levels), and treatment features (use of hypoglycemiants such as insulin, metformin or other drugs). All input variables were transformed into binary data ( 0 or 1$)$, according to the nature of the variable, i.e., qualitative variables were assigned by the absence or presence of the characteristic, and quantitative variables were assigned by values below or above the mean values. The variables used included age, time of disease, gender, serum glucose and glycated hemoglobin values (Hb1Ac), use of insulin, use of metformin, use of any other type of medication, obesity, gestation time (for GDM patients) and number of gestations (for GDM patients) (Figure 1).

\section{Module map gene set variables}

Immune cell specific gene sets were obtained by reanalyzing raw data from the ImmGen project $[44,72]$ (the detailed procedure is provided as Additional file 1). Disease-related gene sets were obtained from GWAS integrator [43] and Phenopedia [42]. GWAS integrator is a compilation of genes offered by GWAS catalogs and databases such as HapMap, SNAP and HugeNavigator [73]. All of the information about genes related to T1D and T2D were considered for use in this study. Phenopedia is an available database about genetic association studies and meta-analysis summarized in the Human Genome Epidemiology (HuGE) encyclopedia [74]. Lists of genes can be obtained about specific diseases or related complications [75]. The available lists of genes related to diabetes mellitus include those associated with pre-diabetic status, diabetes, type 1 diabetes, type 2 diabetes, gestational diabetes, diabetic complications, obesity, angiopathy, ketoacidosis, nephropathy, neuropathy and retinopathy.

\section{Functional analysis}

All clusters of co-regulated genes were analyzed by functional analysis using the Database for Annotation, Visualization, and Integrated Discovery (DAVID) version 6.7 [76]. This approach was used to identify significant biological processes and pathways represented by the differentially expressed genes [77]. A biological process or pathway was considered to be significant if it contained a minimum of three genes per category featuring score values less than 0.05 including the BenjaminiHochberg correction. Moreover, we selected Kyoto Encyclopedia of Genes and Genomes (KEGG) provided by DAVID analysis [76] to explore biological pathways.

\section{Confirmation by real-time PCR}

Relevant and differentially expressed genes obtained using the microarray data were confirmed using qRTPCR (Table 1). The sequences of selected genes were retrieved from the NCBI GenBank database [78]. All major alternative transcripts were considered to design the 
Table 1 Primers used for confirmation by qRT-PCR of the IL1B, RGS1, EGR2, FOXO3A, SOD2 and HIF1A genes

\begin{tabular}{|c|c|c|c|c|}
\hline Gene & Accesion numbers & Primer forward & Primer reverse & Size (bp) \\
\hline IL1B & NM_000576.2 & 5'-CCACAGACCTTCCAGGAGAA-3' & 5'-GTGATCGTACAGGTGCATCG-3' & 121 \\
\hline RGS1 & NM_002922.3 & 5'-TGGCTGGCTTGTGAAGACTA-3' & 5'-GATTCTCGAGTGCGGAAGTC-3' & 131 \\
\hline EGR2 & NM_000399.3 & 5'-GGTGACCATCTITCCCAATG-3' & 5'-TATGGGAGATCCAACGACCT-3' & 120 \\
\hline FОХОЗА & NM_001455.3 & 5'-GTGCTAAGCAGGCCTCATCT-3' & 5'-TTGGCAAAGGGTTTTCTCTG-3' & 119 \\
\hline SOD2 & NM_001024465.1 & 5'-GACAAACCTCAGCCCTAACG-3' & 5'-TTGGACACCAACAGATGCAG-3' & 124 \\
\hline HIF1A & NM_181054.2 & 5'-TCAGCTATTTGCGTGTGAGG-3' & 5'-AAAACCATCCAAGGCTTTCA-3' & 107 \\
\hline GAPDH & NM_002046.3 & 5'-CTCTGCTCCTCCTGTTCGAC-3' & 5'-ACGACCAAATCCGTTGACTC-3' & 112 \\
\hline HPRT1 & NM_000194.2 & 5'-GACCAGTCAACAGGGGACAT-3' & 5'-CTGCATTGTTTTGCCAGTGT-3' & 111 \\
\hline
\end{tabular}

GAPDH and HPRT1 were selected as endogens.

primers, and the Primer3 web tool [79] was used to select pairs of oligonucleotide primers spanning at the exon/exon junction. An optimal melting temperature of $60^{\circ} \mathrm{C}$ was standardized for all genes. Transcriptional expression levels were determined using SYBR Green (Life Technologies), in a StepOne Real Time PCR System (Applied Biosystems). The experiments were done in duplicates. The GAPDH and HPRT1 genes were used as constitutive genes and the selection of the best genes for qRT analysis was done using the Housekeeper software [80]. The standard curve for each primer was determined and only primers with efficiency upper than $90 \%$ were used. It was applied the $\Delta \Delta C T$ method as described by [81], and the minimum expression value was used as calibrator. The graphics were constructed in GraphPad Prism version 6.0 and statistical analysis in the $\mathrm{R}$ statistical environment version 3.0.1. Nonparametric Mann-Whitney $\mathrm{U}$ test was used, considering $P$ value $\leq 0.05$ to be significant.

\section{Conclusion}

The present study revealed that epidemiological, clinical, laboratory, immunological, genetic and treatment features influenced the transcriptome profiles in the major types of diabetes. In addition, inflammation associated with macrophage and dendritic cell function may be responsible for clustering GDM and T1D patients together, while inflammation in T2D may be influenced by drug treatment.

\section{Additional file}

Additional file 1: ImmGen supplementary data.

\section{Competing interests}

The authors declare that they have no competing interests.

\section{Authors' contributions}

Wrote the paper: AFE GAP DP EAD. Conceived the study and participated in its design and coordination: AFE GAP CN DP EAD. Provided material and analytical tools: ETSH GAP CN DP EAD. Performed patient selection, sample collection and cell separation: AFE DJX DMR. Responsible for diabetic patient's treatment and clinical information data: MCFF MFF. Performed RNA extraction and sample quality control: AFE DJX. Responsible for hybridization and acquisition of microarray data: AFE DJX CCVA FSMC CM. Performed data analysis: AFE DP. All authors contributed to and approved the final manuscript.

\section{Acknowledgments}

This study was funded by the Fundação de Amparo à Pesquisa do Estado de São Paulo (FAPESP - (FAPESP \#2008/56594-8, FAPESP \#2010/05622-1, FAPESP \#210/00932-2, FAPESP \#2010/12069-7) and Conselho Nacional de Desenvolvimento Científico e Tecnológico (CNPq \# 563731/2010-9), We thank Dr. Pascal Rihet and the members of TAGC/INSERM-U1090, Marseille, France for the important suggestions. We also thank the ImmGen project members for making available the immune cells raw microarray data.

\section{Author details}

${ }^{1}$ Molecular Immunogenetics Group, Department of Genetics, Faculty of Medicine of Ribeirão Preto, University of São Paulo (USP), 14049-900 Ribeirão Preto, SP, Brazil. 'Division Clinical Immunology, Faculty of Medicine of Ribeirão Preto, (USP), 14049-900 Ribeirão Preto, SP, Brazil. ${ }^{3}$ Department of Biology, Faculty of Philosophy, Sciences and Letters, (USP), 14040-900 Ribeirão Preto, SP, Brazil. ${ }^{4}$ INSERM U1090, TAGC, Aix-Marseille Université IFR137, 13100 Marseille, France. ${ }^{5}$ Disciplines of Genetics and Molecular Biology, Department of Morphology, Physiology and Basic Pathology, School of Dentistry of Ribeirão Preto, USP, 14040-904 Ribeirão Preto, SP, Brazil.

Received: 23 July 2013 Accepted: 27 March 2014

Published: 23 May 2014

\section{References}

1. International Diabetes Federation. In [http://www.idf.org/diabetesatlas/]

2. American Diabetes Association: Diagnosis and classification of diabetes mellitus. Diabetes Care 2011, 34(Suppl 1):S62-S69.

3. Lauenborg J, Hansen T, Jensen DM, Vestergaard H, Mølsted-Pedersen L, Hornnes P, Locht H, Pedersen O, Damm P: Increasing incidence of diabetes after gestational diabetes: a long-term follow-up in a Danish population. Diabetes Care 2004, 27:1194-1199.

4. Bellamy L, Casas J-P, Hingorani AD, Williams D: Type 2 diabetes mellitus after gestational diabetes: a systematic review and meta-analysis. Lancet 2009, 373:1773-1779.

5. Chentoufi AA, Binder NR, Berka N, Abunadi T, Polychronakos C: Advances in type I diabetes associated tolerance mechanisms. Scand J Immunol 2008, 68:1-11.

6. Tisch R, Wang B: Dysregulation of T cell peripheral tolerance in type 1 diabetes. Adv Immunol 2008, 100:125-149.

7. Knip M, Siljander $\mathrm{H}$ : Autoimmune mechanisms in type 1 diabetes. Autoimmun Rev 2008, 7:550-557.

8. Todd JA: Etiology of type 1 diabetes. Immunity 2010, 32:457-467.

9. Sia C: Replenishing peripheral CD4(+) regulatory T cells: a possible immune-intervention strategy in type 1 diabetes? Rev Diabet Stud 2006, 3:102-107.

10. Novak J, Lehuen A: Mechanism of regulation of autoimmunity by iNKT cells. Cytokine 2011, 53:263-270.

11. Cipolletta C, Ryan KE, Hanna EV, Trimble ER: Activation of peripheral blood CD14+ monocytes occurs in diabetes. Diabetes 2005, 54:2779-2786. 
12. Karumuthil-Melethil S, Perez N, Li R, Vasu C: Induction of innate immune response through TLR2 and dectin 1 prevents type 1 diabetes. $\mathrm{J}$ Immunol 2008, 181:8323-8334.

13. Catalog of Published Genome-Wide Association Studies. In [http://www. genome.gov/gwastudies/].

14. Pociot F, Akolkar B, Concannon P, Erlich HA, Julier C, Morahan G, Nierras CR, Todd JA, Rich SS, Nerup J: Genetics of type 1 diabetes: what's next? Diabetes 2010, 59:1561-1571.

15. Plagnol V, Howson JMM, Smyth DJ, Walker N, Hafler JP, Wallace C, Stevens H, Jackson L, Simmonds MJ, Bingley PJ, Gough SC, Todd JA: Genome-wide association analysis of autoantibody positivity in type 1 diabetes cases. PLoS Genet 2011, 7:e1002216.

16. Grinberg-Bleyer Y, Baeyens A, You S, Elhage R, Fourcade G, Gregoire S, Cagnard N, Carpentier W, Tang Q, Bluestone J, Chatenoud L, Klatzmann D, Salomon BL, Piaggio E: IL-2 reverses established type 1 diabetes in NOD mice by a local effect on pancreatic regulatory T cells. J Exp Med 2010, 207:1871-1878.

17. Fornari TA, Donate PB, Macedo C, Sakamoto-Hojo ET, Donadi EA, Passos GA: Development of type 1 diabetes mellitus in nonobese diabetic mice follows changes in thymocyte and peripheral T lymphocyte transcriptional activity. Clin Dev Immunol 2011, 2011:158735.

18. Rassi DM, Junta CM, Fachin AL, Sandrin-Garcia P, Mello S, Silva GL, Evangelista AF, Magalhães DA, Wastowski IJ, Crispim JO, Martelli-Palomino G, Fernandes APM, Deghaide NNHS, Foss-Freitas MC, Foss MC, Soares CP Sakamoto-Hojo ET, Passos GAS, Donadi EA: Gene expression profiles stratified according to type 1 diabetes mellitus susceptibility regions. Ann N Y Acad Sci 2008, 1150:282-289.

19. Han D, Leyva CA, Matheson D, Mineo D, Messinger S, Blomberg BB, Hernandez A, Meneghini LF, Allende G, Skyler JS, Alejandro R, Pugliese A, Kenyon NS: Immune profiling by multiple gene expression analysis in patients at-risk and with type 1 diabetes. Clin Immunol 2011, 139:290-301.

20. Planas R, Pujol-Borrell R, Vives-Pi M: Global gene expression changes in type 1 diabetes: insights into autoimmune response in the target organ and in the periphery. Immunol Lett 2010, 133:55-61.

21. Reynier F, Pachot A, Paye M, Xu Q, Turrel-Davin F, Petit F, Hot A, Auffray C Bendelac N, Nicolino M, Mougin B, Thivolet C: Specific gene expression signature associated with development of autoimmune type-I diabetes using whole-blood microarray analysis. Genes Immun 2010, 11:269-278.

22. Billings LK, Florez JC: The genetics of type 2 diabetes: what have we learned from GWAS? Ann N Y Acad Sci 2010, 1212:59-77.

23. Prokopenko I, McCarthy MI, Lindgren CM: Type 2 diabetes: new genes, new understanding. Trends Genet 2008, 24:613-621.

24. Zeggini E, McCarthy MI: TCF7L2: the biggest story in diabetes genetics since HLA? Diabetologia 2007, 50:1-4

25. Weedon MN, Clark VJ, Qian Y, Ben-Shlomo Y, Timpson N, Ebrahim S, Lawlor DA, Pembrey ME, Ring S, Wilkin TJ, Voss LD, Jeffery AN, Metcalf B, Ferrucci L, Corsi AM, Murray A, Melzer D, Knight B, Shields B, Smith GD, Hattersley AT, Di Rienzo A, Frayling TM: A common haplotype of the glucokinase gene alters fasting glucose and birth weight: association in six studies and population-genetics analyses. Am J Hum Genet 2006, 79:991-1001.

26. Voight BF, Scott $L$, Steinthorsdottir $V$, Morris AP, Dina C, Welch RP, Zeggini E, Huth C, Aulchenko YS, Thorleifsson G, McCulloch LJ, Ferreira T, Grallert H, Amin N, Wu G, Willer CJ, Raychaudhuri S, McCarroll SA, Langenberg C, Hofmann OM, Dupuis J, Qi L, Segrè AV, van Hoek M, Navarro P, Ardlie K, Balkau B, Benediktsson R, Bennett AJ, Blagieva R, et al: Twelve type 2 diabetes susceptibility loci identified through large-scale association analysis. Nat Genet 2010, 42:579-589.

27. Hayashi Y, Kajimoto K, lida S, Sato Y, Mizufune S, Kaji N, Kamiya H, Baba Y, Harashima H: DNA microarray analysis of whole blood cells and insulinsensitive tissues reveals the usefulness of blood RNA profiling as a source of markers for predicting type 2 diabetes. Biol Pharm Bull 2010, 33:1033-1042

28. Ghosh S, Dent R, Harper M-E, Gorman SA, Stuart JS, McPherson R: Gene expression profiling in whole blood identifies distinct biological pathways associated with obesity. BMC Med Genomics 2010, 3:56.

29. Hakonarson H, Grant SFA: GWAS and its impact on elucidating the etiology of diabetes. Diabetes Metab Res Rev 2011, 27:685-696.

30. Macias-Gonzalez M, Cardona F, Queipo-Ortuño M, Bernal R, Martin M, Tinahones FJ: PPARgamma mRNA expression is reduced in peripheral blood mononuclear cells after fat overload in patients with metabolic syndrome. J Nutr 2008, 138:903-907.

31. Ozsari L, Karadurmus N, Sahin M, Uckaya G, Ural AU, Kutlu M: Comparison of lymphomononuclear cell energy metabolism between healthy, impaired glucose intolerance and type 2 diabetes mellitus patients Endocrine 2010, 37:135-139.

32. Kaizer EC, Glaser CL, Chaussabel D, Banchereau J, Pascual V, White PC: Gene expression in peripheral blood mononuclear cells from children with diabetes. J Clin Endocrinol Metab 2007, 92:3705-3711.

33. Kaaja R, Rönnemaa T: Gestational diabetes: pathogenesis and consequences to mother and offspring. Rev Diabet Stud 2008, 5:194-202.

34. Watanabe RM, Black MH, Xiang AH, Allayee $H$, Lawrence JM, Buchanan TA Genetics of gestational diabetes mellitus and type 2 diabetes. Diabetes Care 2007, 30(Suppl 2):S134-S140

35. Robitaille J, Grant AM: The genetics of gestational diabetes mellitus: evidence for relationship with type 2 diabetes mellitus. Genet Med 2008, 10:240-250.

36. Ortega-Senovilla H, Schaefer-Graf U, Meitzner K, Abou-Dakn M, Graf K, Kintscher U, Herrera E: Gestational diabetes mellitus causes changes in the concentrations of adipocyte fatty acid-binding protein and other adipocytokines in cord blood. Diabetes Care 2011, 34:2061-2066.

37. Saucedo R, Zarate A, Basurto L, Hernandez M, Puello E, Galvan R, Campos S: Relationship between circulating adipokines and insulin resistance during pregnancy and postpartum in women with gestational diabetes. Arch Med Res 2011, 42:318-323.

38. Radaelli T, Lepercq J, Varastehpour A, Basu S, Catalano PM, Hauguel-De Mouzon S: Differential regulation of genes for fetoplacental lipid pathways in pregnancy with gestational and type 1 diabetes mellitus. Am J Obstet Gynecol 2009, 201:209.e1-209.e10.

39. Zhao Y-H, Wang D-P, Zhang L-L, Zhang F, Wang D-M, Zhang W-Y: Genomic expression profiles of blood and placenta reveal significant immune-related pathways and categories in Chinese women with gestational diabetes mellitus. Diabet Med 2011, 28:237-246.

40. Collares CVA, Evangelista AF, Xavier DJ, Takahashi P, Almeida R, Macedo C, Manoel-Caetano F, Foss MC, Foss-Freitas MC, Rassi DM, Sakamoto-Hojo ET, Passos GA, Donadi EA: Transcriptome meta-analysis of peripheral lymphomononuclear cells indicates that gestational diabetes is closer to type 1 diabetes than to type 2 diabetes mellitus. Mol Biol Rep 2013, 40:5351-5358.

41. Segal E, Friedman N, Koller D, Regev A: A module map showing conditional activity of expression modules in cancer. Nat Genet 2004, 36:1090-1098

42. HuGENavigator|PhenoPedia|Search. In [http://www.hugenavigator.net/ HuGENavigator/startPagePhenoPedia.do]

43. HuGENavigator|GWAS Integrator|Search. In [http://www.hugenavigator net/HuGENavigator/gWAHitStartPage.do].

44. Immunological Genome Project. In [http://www.immgen.org/]

45. Tannahill GM, O'Neill LAJ: The emerging role of metabolic regulation in the functioning of Toll-like receptors and the NOD-like receptor Nlrp3. FEBS Lett 2011, 585:1568-1572.

46. Wu C-H, Yeh C-T, Shih P-H, Yen G-C: Dietary phenolic acids attenuate multiple stages of protein glycation and high-glucose-stimulated proinflammatory IL-1 beta activation by interfering with chromatin remodeling and transcription in monocytes. Mol Nutr Food Res 2010 54(Suppl 2):S127-S140.

47. Miao L, St Clair DK: Regulation of superoxide dismutase genes: implications in disease. Free Radic Biol Med 2009, 47:344-356.

48. Savic D, Ye H, Aneas I, Park S-Y, Bell Gl, Nobrega MA: Alterations in TCF7L2 expression define its role as a key regulator of glucose metabolism. Genome Res 2011, 21:1417-1425.

49. Cantó C, Gerhart-Hines Z, Feige JN, Lagouge M, Noriega L, Milne JC, Elliott PJ, Puigserver $P$, Auwerx J: AMPK regulates energy expenditure by modulating NAD + metabolism and SIRT1 activity. Nature 2009, 458:1056-1060.

50. Cheng K, Ho K, Stokes R, Scott C, Lau SM, Hawthorne WJ, O'Connell PJ, Loudovaris T, Kay TW, Kulkarni RN, Okada T, Wang XL, Yim SH, Shah Y, Grey ST, Biankin AV, Kench JG, Laybutt DR, Gonzalez FJ, Kahn CR, Gunton JE: Hypoxia-inducible factor-1alpha regulates beta cell function in mouse and human islets. J Clin Invest 2010, 120:2171-2183.

51. Fernandes APM, Louzada-Junior P, Foss MC, Donadi EA: HLA-DRB1, DQB1, and DQA1 allele profile in Brazilian patients with type 1 diabetes mellitus. Ann N Y Acad Sci 2002, 958:305-308.

52. Van Belle TL, Coppieters KT, von Herrath MG: Type 1 diabetes: etiology, immunology, and therapeutic strategies. Physiol Rev 2011, 91:79-118.

53. Törn C, Gupta M, Sanjeevi CB, Aberg A, Frid A, Landin-Olsson M: Different HLA-DR-DQ and MHC class I chain-related gene A (MICA) genotypes in 
autoimmune and nonautoimmune gestational diabetes in a Swedish population. Hum Immunol 2004, 65:1443-1450.

54. Fernandes APM, Foss MC, Donadi EA: HLA-DQB1 alleles may influence the surface expression of DQ molecules in lymphomononuclear cells of type 1 diabetes mellitus patients. Scand J Immunol 2004, 59:305-309.

55. Williams AJK, Aitken RJ, Chandler MA-M, Gillespie KM, Lampasona V, Bingley PJ: Autoantibodies to islet antigen- 2 are associated with HLA-DRB1*07 and DRB $1{ }^{*} 09$ haplotypes as well as DRB $1{ }^{*} 04$ at onset of type 1 diabetes: the possible role of HLA-DQA in autoimmunity to IA-2. Diabetologia 2008 51:1444-1448.

56. Papadopoulou A, Lynch KF, Shaat N, Nilsson A, Lernmark B, Berntorp K, Ivarsson S-A, Agardh C-D, Lernmark A: The type 1 diabetes protective HLA DQB $1{ }^{*} 0602$ allele is less frequent in gestational diabetes mellitus. Diabetologia 2009, 52:1339-1342.

57. Lauenborg J, Grarup N, Damm P, Borch-Johnsen K, Jørgensen T, Pedersen O, Hansen T: Common type 2 diabetes risk gene variants associate with gestational diabetes. J Clin Endocrinol Metab 2009, 94:145-150.

58. Wong FS, Hu C, Xiang Y, Wen L: To B or not to B-pathogenic and regulatory B cells in autoimmune diabetes. Curr Opin Immunol 2010, 22:723-731.

59. Song J, Ren $\mathrm{P}$, Zhang L, Wang XL, Chen L, Shen $\mathrm{YH}$ : Metformin reduces lipid accumulation in macrophages by inhibiting FOXO1-mediated transcription of fatty acid-binding protein 4. Biochem Biophys Res Commun 2010, 393:89-94.

60. ArrayExpress < EMBL-EBI. In [http://www.ebi.ac.uk/arrayexpress/]

61. The R Project for Statistical Computing. In [http://www.r-project.org/]

62. AgiND. In [http://tagc.univ-mrs.fr/tagc/index.php/software/].

63. TAGC - INSERM U928 - AgiND. In [http://tagc.univ-mrs.fr/welcome/spip. php?rubrique194].

64. Bioconductor - RTools4TB. In [http://www.bioconductor.org/packages/2.5/ bioc/html/RTools4TB.html].

65. Lopez F, Textoris J, Bergon A, Didier G, Remy E, Granjeaud S, Imbert J, Nguyen C, Puthier D: TranscriptomeBrowser: a powerful and flexible toolbox to explore productively the transcriptional landscape of the gene expression omnibus database. PLoS One 2008, 3:e4001.

66. R: Principal Components Analysis. In [http://stat.ethz.ch/R-manual/Rpatched/library/stats/html/prcomp.html]

67. CRAN - Package rgl. In [http://cran.r-project.org/web/packages/rgl/index.html].

68. Bioconductor - RankProd. In [http://www.bioconductor.org/packages/ release/bioc/html/RankProd.html].

69. Hong F, Breitling R, McEntee CW, Wittner BS, Nemhauser JL, Chory J: RankProd: a bioconductor package for detecting differentially expressed genes in meta-analysis. Bioinformatics 2006, 22:2825-2827.

70. EisenLab. In [http://rana.lbl.gov/EisenSoftware.htm]

71. Eisen MB, Spellman PT, Brown PO, Botstein D: Cluster analysis and display of genome-wide expression patterns. Proc Natl Acad Sci U S A 1998, 95:14863-14868.

72. Immunological Genome Project (ImmGen). In [http://www.Immgen.org/ index_content.html].

73. Yu W, Yesupriya A, Wulf A, Hindorff LA, Dowling N, Khoury MJ, Gwinn M: GWAS integrator: a bioinformatics tool to explore human genetic associations reported in published genome-wide association studies. Eur J Hum Genet 2011, 19:1095-1099.

74. HuGENavigator. In [http://www.hugenavigator.net/HuGENavigator/home.do].

75. Yu W, Clyne M, Khoury MJ, Gwinn M: Phenopedia and genopedia: disease-centered and gene-centered views of the evolving knowledge of human genetic associations. Bioinformatics 2010, 26:145-146.

76. DAVID Functional Annotation Bioinformatics Microarray Analysis. In [http://david.abcc.ncifcrf.gov/].

77. Huang DW, Sherman BT, Tan Q, Kir J, Liu D, Bryant D, Guo Y, Stephens R, Baseler MW, Lane HC, Lempicki RA: DAVID bioinformatics resources: expanded annotation database and novel algorithms to better extract biology from large gene lists. Nucleic Acids Res 2007, 35:W169-W175.
78. GenBank Home. In [http://www.ncbi.n/m.nih.gov/genbank/].

79. Primer3 Input \$CGI_VERSION. In [http://biotools.umassmed.edu/bioapps/ primer3_www.cgi]

80. Pfaffl MW, Tichopad A, Prgomet C, Neuvians TP: Determination of stable housekeeping genes, differentially regulated target genes and sample integrity: BestKeeper-excel-based tool using pair-wise correlations. Biotechnol Lett 2004, 26:509-515.

81. Pfaffl MW: A new mathematical model for relative quantification in real-time RT-PCR. Nucleic Acids Res 2001, 29:e45.

doi:10.1186/1755-8794-7-28

Cite this article as: Evangelista et al: Integrative analysis of the transcriptome profiles observed in type 1, type 2 and gestational diabetes mellitus reveals the role of inflammation. BMC Medical Genomics 2014 7:28.

\section{Submit your next manuscript to BioMed Central and take full advantage of:}

- Convenient online submission

- Thorough peer review

- No space constraints or color figure charges

- Immediate publication on acceptance

- Inclusion in PubMed, CAS, Scopus and Google Scholar

- Research which is freely available for redistribution

Submit your manuscript at www.biomedcentral.com/submit
C Biomed Central 